\title{
Neurodevelopmental Trajectories of the Human Cerebral Cortex
}

\author{
Philip Shaw, ${ }^{1}$ Noor J. Kabani, ${ }^{3}$ Jason P. Lerch, ${ }^{4}$ Kristen Eckstrand, ${ }^{1}$ Rhoshel Lenroot, ${ }^{1}$ Nitin Gogtay, ${ }^{1}$ \\ Deanna Greenstein, ${ }^{1}$ Liv Clasen, ${ }^{1}$ Alan Evans, ${ }^{4}$ Judith L. Rapoport, ${ }^{1}$ Jay N. Giedd, ${ }^{1}$ and Steve P. Wise ${ }^{2}$ \\ ${ }^{1}$ Child Psychiatry Branch and ${ }^{2}$ Laboratory of Systems Neuroscience, National Institute of Mental Health, Bethesda, Maryland 20892, ${ }^{3}$ Sunnybrook Health \\ Sciences Centre, Toronto, Ontario, Canada M4N 3N1, and ${ }^{4}$ Montreal Neurological Institute, McGill University, Montreal, Quebec, Canada H3A 2B4
}

Understanding the organization of the cerebral cortex remains a central focus of neuroscience. Cortical maps have relied almost exclusively on the examination of postmortem tissue to construct structural, architectonic maps. These maps have invariably distinguished between areas with fewer discernable layers, which have a less complex overall pattern of lamination and lack an internal granular layer, and those with more complex laminar architecture. The former includes several agranular limbic areas, and the latter includes the homotypical and granular areas of association and sensory cortex. Here, we relate these traditional maps to developmental data from noninvasive neuroimaging. Changes in cortical thickness were determined in vivo from 764 neuroanatomic magnetic resonance images acquired longitudinally from 375 typically developing children and young adults. We find differing levels of complexity of cortical growth across the cerebrum, which align closely with established architectonic maps. Cortical regions with simple laminar architecture, including most limbic areas, predominantly show simpler growth trajectories. These areas have clearly identified homologues in all mammalian brains and thus likely evolved in early mammals. In contrast, polysensory and high-order association areas of cortex, the most complex areas in terms of their laminar architecture, also have the most complex developmental trajectories. Some of these areas are unique to, or dramatically expanded in primates, lending an evolutionary significance to the findings. Furthermore, by mapping a key characteristic of these development trajectories (the age of attaining peak cortical thickness) we document the dynamic, heterochronous maturation of the cerebral cortex through time lapse sequences ("movies").

Key words: brain development; cytoarchitecture; cognition; cerebral cortex; prefrontal cortex; primate

\section{Introduction}

Most maps of human cerebral cortex have partitioned it according to histological features, such as the distribution of cell bodies or myelin, and, more recently, molecular markers (von Economo and Koskinas, 1925; Ongur et al., 2003; Zilles et al., 2004). Comparisons of similar classifications among several species provide an evolutionary perspective, and such analyses have identified two broad cortical types. One type, allocortex, has a primitive three-layered form that strongly resembles its homologues in reptiles. Another type, isocortex, lacks such homologues and has a more derived structure characterized by more than three layers (typically six) and a more complex pattern of afferent and efferent projections (Kaas, 1987; Puelles, 2001; Allman et al., 2002; Striedter, 2005). Between allocortex and isocortex, areas sometimes called "transition cortex" have intermediate properties. Using structural neuroimaging of the in vivo developing brain, we explored the possibility that these different kinds of cortex show

\footnotetext{
Received Nov. 30, 2007; revised Feb. 7, 2008; accepted Feb. 26, 2008.

This work was supported by the Intramural Research Program of the National Institutes of Health. We thank all the participants in this study and their families.

The authors declare no competing financial interests.

Correspondence should be addressed to Philip Shaw, Child Psychiatry Branch, Room 3N202, Building 10, Center Drive, National Institute of Mental Health, Bethesda, MD 20892. E-mail: shawp@mail.nih.gov.

D01:10.1523/JNEUROSCI.5309-07.2008

Copyright $\odot 2008$ Society for Neuroscience $\quad 0270-6474 / 08 / 283586-09 \$ 15.00 / 0$
}

differing levels of complexity in the trajectories of their growth in childhood and adolescence.

Using computational neuroanatomy, we defined cortical thickness at over 40,000 points throughout the cerebrum in a cohort of 375 healthy children and adolescents. Cortical thickness was chosen as a metric which both captures the columnar architecture of the cortex and is sensitive to developmental change in typically developing and clinical populations (Lerch et al., 2005; O'Donnell et al., 2005; Makris et al., 2006; Shaw et al., 2006a,b; Lu et al., 2007; Sowell et al., 2007).

Most of the children in our cohort had repeated neuroanatomic imaging, and such longitudinal data can be combined with cross-sectional data to model developmental change, with the longitudinal data being particularly informative. For cortical thickness, the simplest trajectory that can be fitted to describe its change over time is a straight line. More complex growth models include distinct phases of increase and decrease in cortical thickness: a quadratic model has two such phases (typically an initial increase which reaches a peak before declining) and a cubic model has three. The complexity of growth may vary across the cerebral cortex, and we sought to explore whether this variation aligned with cytoarchitectural properties.

Derived properties of developmental curves, such as the age of reaching various points of inflection, are frequently used as developmental indices (Tanner et al., 1976; Jolicoeur et al., 1988). 


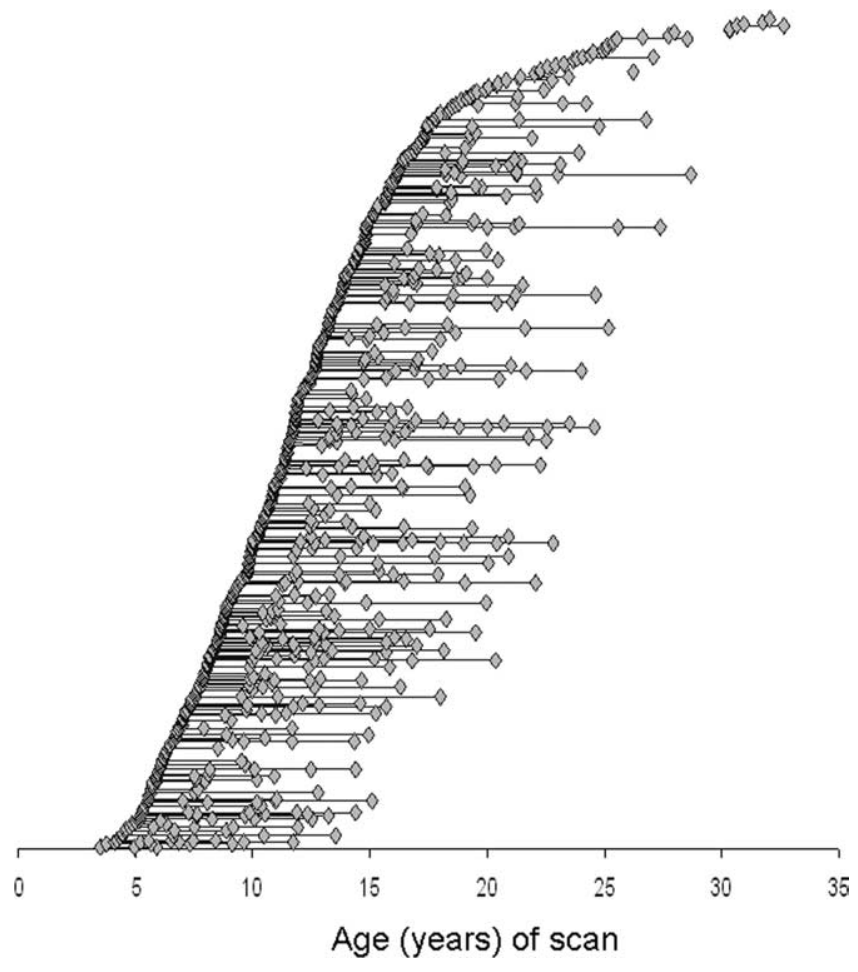

Figure 1. The age distribution of the data. The age at each scan is indicated by a blue diamond. For each subject, the first scan is always the leftmost; subjects with repeated scans have a horizontal line drawn connecting the age at first scan with the age at later scans.

For cortical thickness, the age at which peak cortical thickness is reached (the point where increase gives way to decrease in cortical thickness) can be determined for cortical points with either a cubic or quadratic (but not linear) trajectory and, thus, emerges as a potentially useful index of cortical development. We therefore additionally examined the pattern of attaining peak cortical thickness across the cerebrum, to confirm and expand previous observations of a heterochronous sequence, with primary sensory areas attaining peak cortical thickness before polymodal, high-order association areas (Gogtay et al., 2004).

\section{Materials and Methods}

Participants. Three hundred and seventy-five children and adolescents, healthy children with no personal or family history of psychiatric or neurological disorders, had a total of 764 magnetic resonance images. Each subject completed the Childhood Behavior Checklist as a screening tool and then underwent a structured diagnostic interview by a child psychiatrist to rule out any psychiatric or neurological diagnoses (Giedd et al., 1996). Handedness was determined using the PANESS (Physical and Neurological Examination for Soft Signs), and 336 (90\%) were predominately right-handed, 20 (5\%) predominately left-handed, and 19 (5\%) ambidextrous. The mean intelligence quotient (IQ) was 115 (SD, 13) as determined from age appropriate versions of the Wechsler Intelligence Scales (Shaw et al., 2006b). Socioeconomic status (SES) was determined from the Hollingshead Scales and the mean score was 40 (SD, 19) (Hollingshead, 1975). The age range spanned from 3.5 to 33 years, and the age distribution of scans is illustrated in Figure 1. The subjects came from 292 different families; 196 (52\%) were male. The age range spanned from 3.5 to 33 years. All subjects had at least one scan (mean age at initial scan, 12.3 years; SD, 5.3); $203(54.1 \%)$ had at least two scans (mean age, 13.8; SD, 4.6); $106(28.3 \%)$ had at least three scans (mean, 15.3 ; SD , 4.2); and 57 (15.2\%) had four or more scans (mean 18, SD 4.5).

Neuroimaging. T1-weighted images with contiguous $1.5 \mathrm{~mm}$ axial slices and $2.0 \mathrm{~mm}$ coronal slices were obtained using three-dimensional spoiled gradient recalled echo in the steady state on a 1.5-T General
Electric (Milwaukee, WI) Signa scanner. Imaging parameters were as follows: echo time, $5 \mathrm{~ms}$; repetition time, $24 \mathrm{~ms}$; flip angle, $45^{\circ}$; acquisition matrix, $256 \times 192$; number of excitations, 1 ; and field of view, $24 \mathrm{~cm}$ Head placement was standardized as described previously (Giedd et al., 1999). The same scanner was used throughout the study. The native MRI scans were registered into standardized stereotaxic space using a linear transformation and corrected for nonuniformity artifacts (Sled et al., 1998). The registered and corrected volumes were segmented into white matter, gray matter, CSF, and background using an advanced neural net classifier (Zijdenbos et al., 2002). A surface deformation algorithm was applied which first fits the white matter surface, then expands outward to find the gray matter-CSF intersection defining a known relationship between each vertex of the white matter surface and its gray matter surface counterpart; cortical thickness is defined as the distance between these linked vertices (and measured at 40,962 such vertices) (MacDonald et al., 2000). A 30 -mm-bandwidth blurring kernel was applied; this size was chosen on the basis of population simulations which that this bandwidth maximized statistical power while minimizing false positives (Lerch and Evans, 2005). This kernel allows anatomical localization, as $30 \mathrm{~mm}$ blurring along the surface using a diffusion smoothing operator preserves cortical topologic features and represents considerably less cortex than the equivalent volumetric Gaussian blurring kernel.

The validity of this automated measure against expert manual neuroanatomical estimation of cortical thickness has been demonstrated previously for selected cortical regions in an adult population (Kabani et al., 2001) We repeated this validation study in our pediatric population in the cortical regions included in the original study (the pre and post central gyri, the superior frontal gyrus, the superior temporal gyrus, the cuneus, the superior parietal lobule and supramarginal gyrus) (Kabani et al., 2001). We also examined regions of particular interest for this study. These were the insula, the orbitofrontal cortex (measured bilaterally in its anterior, posterior, medial, and lateral divisions), and medial cortical regions (the anterior and posterior cingulate, the medial dorsal prefrontal cortex, and the parahippocampal gyrus). Twenty scans were chosen at random from the cohort (from ages 6 through 15). For each brain region the neuroanatomist (N.K.) used image analyses software (MacDonald, 1996) to mark one point or tag on the CSF and gray matter border which represents the outer surface of the cortex, and another point of the gray and white matter border which represents the inner surface of the cortex. The distance between the two tags was calculated, mimicking the algorithm used by the automated tool. For a given tag placed by the neuroanatomist on the outer cortical surface, the closest vertex on the automatically extracted cortical surface was identified and its associated cortical thickness was noted. The output of the manual and automatic methods were compared using a repeated measures ANOVA followed by paired $t$ tests to identify regional differences. There was a significant difference for type of measurement with the automated estimates being larger (mean, 4.62; SE, 0.06) than the manual (mean, 4.41; SE, 0.04; $\left.F_{(1,684)}=8.8, p=0.02\right)$. There was a significant interaction of the type of measurement and region $\left(F_{(35,684)}=2.59, p<0.001\right)$ which was further explored. Overall there was no significant difference between the manual and automated measures in 30 of the 36 regions, with poorer performance noted bilaterally in the precentral gyrus, and in the left postcentral gyrus, and in the middle frontal gyrus, gyrus rectus and the cuneus in the left hemisphere. Notably, only one of these regions lay in an area of particular interest for this study (the left gyrus rectus). There was no correlation between age and the difference between the automated and manual estimates $(r=0.02, p=0.53)$. Thus, there was no evidence that the differences between the two metrics had any significant age related bias.

To determine developmental trajectories at each cortical point, mixed model regression analysis was chosen as it permits the inclusion of multiple measurements per person, missing data, and irregular intervals between measurements, thereby increasing statistical power (Pinheiro and Bates, 2000). Our classification of developmental trajectories was based on a step-down model selection procedure: at each cortical point we modeled cortical thickness using a mixed-effects polynomial regression model, testing for cubic, quadratic and linear age effects. If the cubic age effect was not significant at $p<0.05$, it was removed and we stepped 
down to the quadratic model and so on. In this way, we were able to classify the development of each cortical point as being best explained by a cubic, quadratic, or linear function of age. We consider cubic models to be more complex than quadratic, which in turn are held to be more complex than linear models. A random effect for each individual was nested within a random effect for each family, thus accounting for both within-person and within-family dependence. Thus, for cortical points with a cubic model, the $k$ th cortical thickness of the $i$ th individual in the $j$ th family was modeled as thickness ${ }_{i j k}=$ intercept $+d_{i j}+\beta_{1}$ (age $)+\beta 2^{\star}(\text { age })^{\star *} 2+$ $\beta 3^{\star}(\text { age })^{\star *} 3+e_{i j k}$, where $d_{i j}$ are nested random effects modeling within-person and within family dependence, the intercept and $\beta$ terms are fixed effects, and $e_{i j k}$ represents the residual error. Quadratic models lacked the cubic age term, and linear models the cubic and quadratic age terms. The analyses were repeated entering SES and IQ as covariates.

The age at which peak cortical thickness was attained was calculated for cubic and quadratic models from the first order derivatives of the fitted curves.

\section{Results}

Throughout most of the lateral frontal, lateral temporal, parietal and occipital isocortex, developmental trajectories are cubic, with a period of initial childhood increase, followed by adolescent decline and then stabilization of cortical thickness in adulthood (Fig. 2). Growth characterized by increase and decrease, but lacking the phase of stabilization within the first three decades of life (a quadratic model) is present in much of the insula and anterior cingulate cortex. A linear trajectory is seen in the posterior orbitofrontal and frontal operculum, portions of the piriform cortex, the medial temporal cortex, subgenual cingulate areas, and medial occipitotemporal cortex. Graphs illustrating individual data points from representative regions with a cubic, quadratic or linear trajectory are shown in Figure 3.

We examined the complexity of developmental trajectories with respect to cortical regions of differing cytoarchitecturonic types, using histological atlases to assign cytoarchitectonic fields (Ongur et al., 2003). This analysis revealed a clear parallel between basic types of cortex and the pattern of cortical development. The orbitofrontal cortex exemplifies the correspondence between cortical types and developmental trajectories (Fig. 4). In the most anterior part of this region, a cubic trajectory characterizes the homotypical (six-layered) isocortex of the frontal pole and lateral orbitofrontal regions. In contrast, most of the cortex on the posterior orbital surface follows relatively simple quadratic and linear growth trajectories. This region has a lamination pattern typical of transitional cortex: compared with homotypical isocortex it has fewer, less well developed layers and lacks the clear concentration of nonpyramidal cells of layer 4, the internal granular layer (Brockhaus, 1940; Mesulam and Mufson, 1982; Ongur et al., 2003). In the most posterior part of this region, out.
QUADRATIC

LINEAR
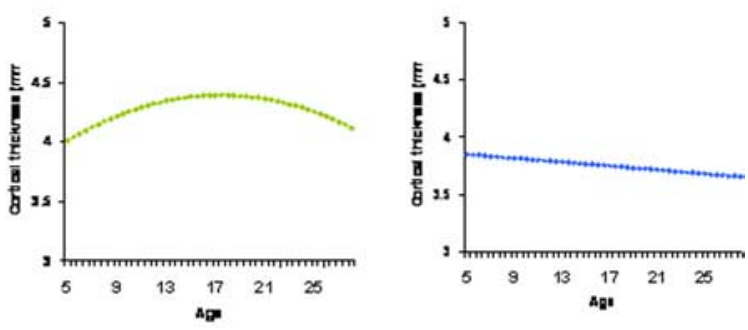

Figure 2. Complexity of developmental trajectories throughout the cerebral cortex. The brain maps show the vertices having a cubic (red), quadratic (green) or linear (blue) developmental trajectory. The graphs show the growth pattern for each of these divisions. In order there are dorsal, right lateral, left medial, left lateral, and right medial views. The corpus callosum is blacked

linear and quadratic growth characterizes the piriform cortex, a primitive allocortical area that subserves olfaction.

Although Figure 4 focuses on the orbitofrontal cortex, the same principles are observed generally, where a transition from isocortex to simpler forms occurs. The results for the medial frontal cortex are similar to those in the orbitofrontal cortex, with cubic growth anteriorly, especially in the homotypical cortex of the medial frontal pole- and linear or quadratic trajectories more posteriorly in regions of dysgranular or agranular architecture (Fig. 5, top). For the insula (Fig. 5, bottom), the pattern is much the same. The anterior insula, with its agranular and poorly laminated cortex, has a linear developmental trajectory. Moving posteriorly to the dysgranular and homotypical insula, there is at first a more complex quadratic fit; still more posteriorly, as the cortex becomes increasingly homotypical, the trajectory becomes cubic. Likewise in the temporal lobe, an allocortical component such as the piriform cortex shows a predominately a linear trajectory. In contrast, the lateral temporal isocortex has a cubic trajectory and transition areas such as the entorhinal and perirhinal regions have quadratic and linear trajectories (Fig. 2). These results are 
(a) Superior frontal gyri (cubic)

\section{(b) Insula (quadratic)}

\section{(c) Orbitofrontal} gyri (linear)
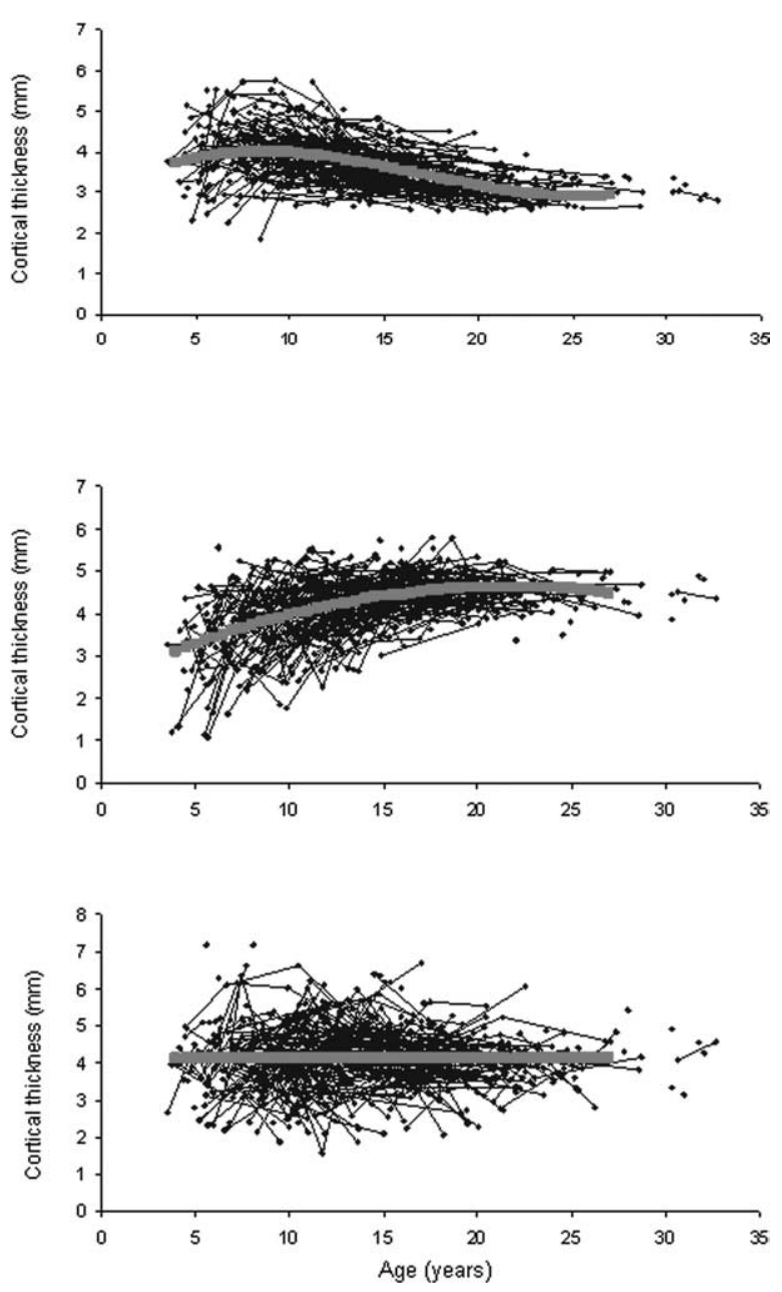

Figure 3. Graphs showing raw cortical thickness data in blue, with the fitted trajectory superimposed in pink. $\boldsymbol{a}-\boldsymbol{c}$, The first three images show in order the mean cortical thickness and trajectory for representative regions: the superior frontal gyri, which have a cubic trajectory $(\boldsymbol{a})$; the portion of the insula which has a quadratic trajectory, seen in green in Figure $5(\boldsymbol{b})$; the portion of the orbitofrontal cortex which has a linear trajectory, seen in blue in Figure 4 (c).

summarized in Table 1. There are some cortical regions where this link between cortical types and developmental trajectories does not hold, most notably in the medial occipitotemporal and the anterior superior temporal areas, both of which are isocortical regions that nonetheless have a linear and quadratic trajectory, respectively. The pattern of results held when SES and IQ were entered as covariates, either separately or together.

We next determined the age at which peak cortical thickness was attained for all points with either a cubic or quadratic trajectory, using the first order derivative of the fitted curve for each point. A peak age cannot be determined for the points with a linear trajectory. The results are presented as a time-lapse dynamic sequence (supplemental Movies 1, 2, available at www. jneurosci.org as supplemental material), "stills" taken from the movies (Fig. 6), and the estimated age of peak cortical thickness for 56 brain regions (as defined by the ANIMAL segmentation tool).

To summarize the results, within isocortex, the primary sensory and motor areas generally attain their peak cortical thickness before adjacent secondary areas, and also before other polymodal association areas. In the posterior brain, the first area to reach its peak thickness is the somatic sensory cortex ( $\sim 7$ years $)$, followed by the occipital poles, containing much of the striate primary visual area $(\sim 7$ years on the left, and $\sim 8$ years on the right) and then the remaining parieto-occipital cortex, with polymodal regions (such as the posterior parietal cortex) reaching peak thickness later ( $\sim 9-10$ years). In the frontal cortex, the primary motor cortex attains peak cortical thickness relatively early $(\sim 9$ years), followed by the supplementary motor areas ( $\sim 10$ years $)$ and most of the frontal pole $(\sim 10$ years $)$. High-order cortical areas, such as the dorsolateral prefrontal cortex and cingulate cortex, reach peak thickness last ( $\sim 10.5$ years $)$. In the medial views, the occipital and frontal poles attain peak thickness early, and then a centripetal wave sweeps from these areas, with the medial prefrontal and cingulate cortex attaining peak thickness last. There is also a marked dorsal to ventral progression of development. Detailed results for each brain regions are given in Table 2 .

\section{Discussion \\ Alignment of cortical types with developmental trajectories}

This study demonstrates a tight alignment between developmental trajectories and the cortical types depicted in traditional cytoarchitectonic maps, affording these classic maps a developmental significance. The study both supports and extends previous work (Gogtay et al., 2004; Sowell et al., 2004; O’Donnell et al., 2005) through the inclusion of a much larger sample size which allowed the detection of higher order effects of age.

Other longitudinal studies of typical development support some of the present findings. For example, the simple linear growth we report in one part of allocortex, the piriform area, has also been found previously for the hippocampus (Gogtay et al., 2006). We were unable to measure the hippocampus directly in the present study, but Gogtay and colleagues found that the trajectory of volume change of the allocortical hippocampus was linear. The prominence of isocortical thinning in adolescence is confirmed in studies using other cortical morphometric measures such as gray matter density, demonstrating the complementary nature of these different measures of cortical characteristics (Gogtay et al., 2004; Sowell et al., 2004).

The model used here applies only to the age range covered and cannot be extrapolated. For example, if the cubic trajectory were extended beyond the age range, it would imply there is an increase in cortical thickness in adulthood (starting around the age of $\sim 25$ ), which is neither biologically plausible nor supported by existing data in this age range (Sowell et al., 2007). Rather, the age at which the phase of cortical thinning stops (the second point of inflection in a cubic curve) is better conceptualized as the points of transition into the essentially stable cortical dimensions of adulthood. Areas with cubic trajectories reach this inflection point faster than those with quadratic curves, and in this sense could be conceptualized as having faster growth. 


\section{Methodological issues}

It is important to consider the possibility of methodological artifact contributing to the results. This could arise, for example, as the cortical surface reconstruction, which is the basis of the automated technique, may be particularly difficult in regions of the allocortex and transitional cortex, and the resulting increase in measurement error might obscure complex (cubic) growth patterns. Several factors make this unlikely. The validity of the cortical thickness measure judged against manual estimates did not vary systematically with type of cortex. The automated cortical thickness estimates in allocortical regions of the orbitofrontal and medial cortex were as valid as the measurements of isocortical regions. Additionally, the algorithm we used and its derivatives can also accurately extract the cortical surfaces of a "phantom" brain, detect simulated thinning of the cortex and capture the neuropathologically established patterns of disease progression (MacDonald et al., 2000; Lerch and Evans, 2005; Lerch et al., 2005; Lee et al., 2006). Finally, our study benefits from its large sample size and high proportion of prospective data, factors which afforded the detection of linear and curvilinear effects of age on cortical growth which were both statistically and, we argue biologically, significant.

\section{Environmental and genetic effects on growth trajectories}

The nature of the cellular events underpinning cortical change in humans has not been established. Some of the earliest aspects of cortical development, such as the emergence and resolution of the subplate as neuroblasts migrate from the neuroepithelium to their mature laminar locations (Kostovic and Rakic, 1990; Kostovic et al., 2002) determine cerebral lamination in utero and perinatally, but fall outside the age window we studied. Studies in nonhuman animal suggest that cortical dimensions during critical periods for the development of cognitive functions may reflect experience-dependent molding of the architecture of cortical columns along with dendritic spine and axonal remodeling (Chklovskii et al., 2004; Mataga et al., 2004; Hensch, 2005; Sur and Rubenstein, 2005). Such morphological events may contribute to the childhood phase of increase in cortical thickness, which occurs in regions with either a cubic or quadratic trajectory. The phase of cortical thinning which dominates adolescence might reflect the use-dependent selective elimination of synapses (Huttenlocher and Dabholkar, 1997) that could refine neural circuits, including those supporting cognitive abilities (Hensch, 2004; Knudsen, 2004). Events occurring at the interface between white
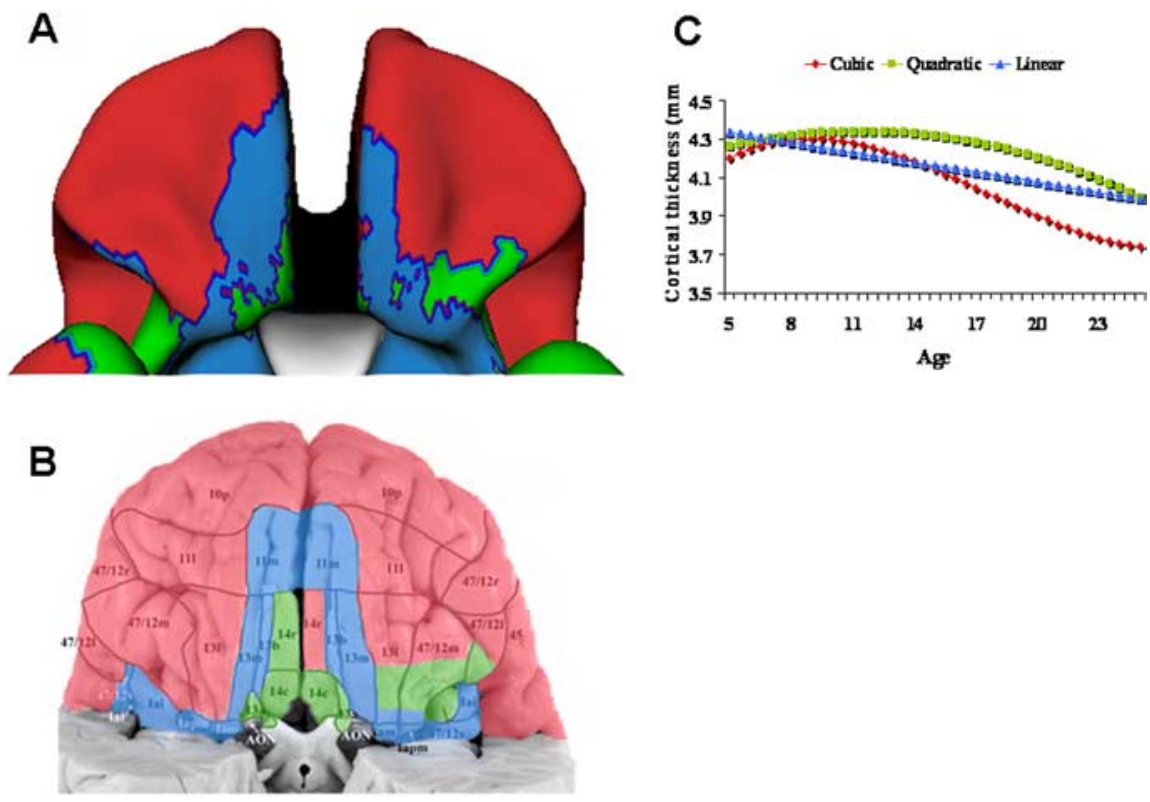

Figure 4. $A$, Complexity of developmental trajectories throughout the orbitofrontal cortex, projected onto a standard brain template. The anterior and lateral orbitofrontal cortex has a cubic fit (red); medial and posterior orbitofrontal cortex has simpler quadratic (green) and linear (blue) trajectories. $\boldsymbol{B}$, The trajectories are superimposed on a cytoarchitectonic map of the region by Öngür et al. (2003) to illustrate the overlap between the cytoarchitectonic fields and regional differences in trajectories. $\boldsymbol{C}$, The trajectory of each of the divisions.
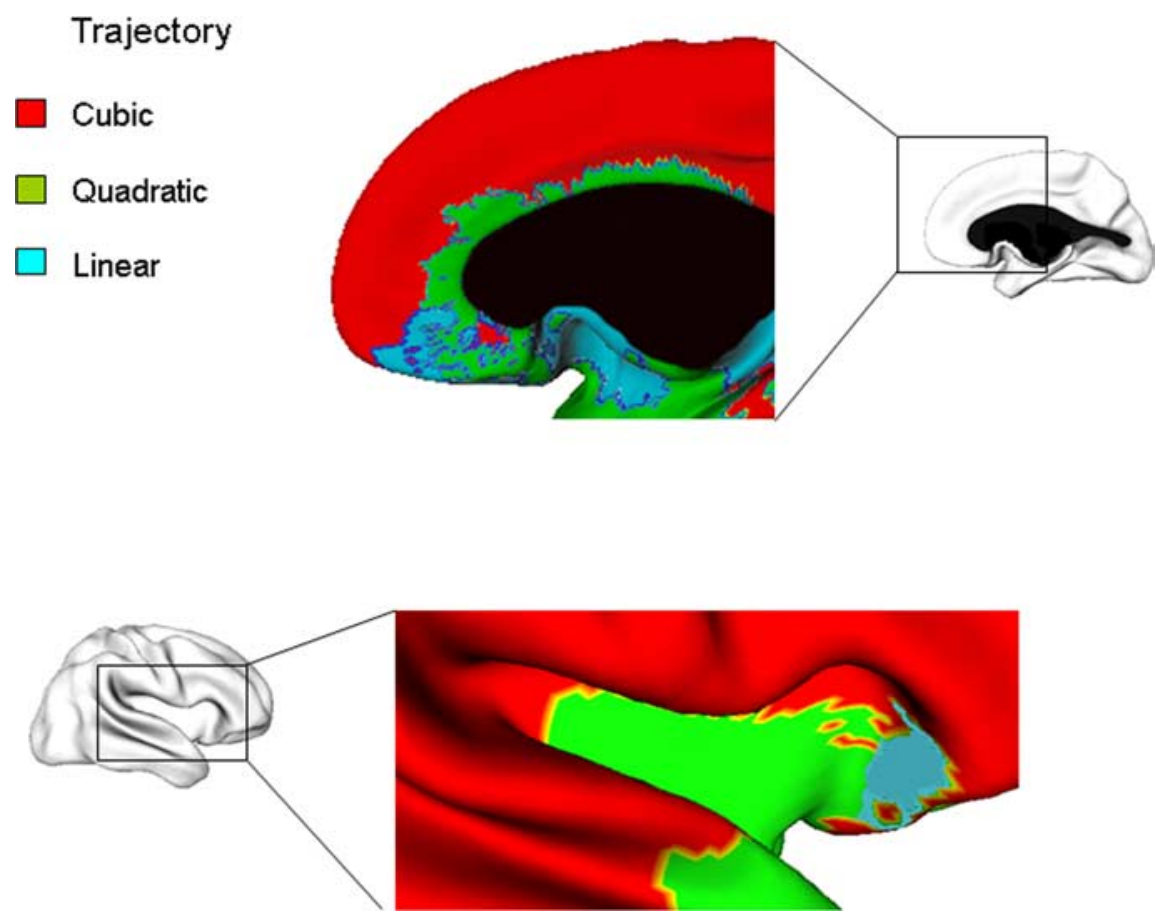

Figure 5. Top, Detailed views of trajectories in the right medial prefrontal cortex, where isocortical regions have a cubic trajectory, and transitional areas have either a quadratic trajectory (e.g., the agranular and poorly laminated cortex of area 24a in the cingulate gyrus) or a linear decline in thickness (e.g., the thin and largely agranular cortex of the gyrus rectus). Bottom, The right insula shows progressively more complex trajectories moving: the posterior portion has a cubic trajectory (red), the body of the insula has a quadratic fit (green) and the anterior insula has a linear fit (blue). A similar pattern holds for the left insula.

and gray matter, such as the proliferation of myelin into the peripheral cortical neuropil in childhood and adolescence, may also influence cortical thickness (Yakovlev and Lecours, 1967; Sowell et al., 2004).

This account of possible cellular events emphasizes the role of 
Table 1. The different orders of trajectories are given with the corresponding cortical regions and the underlying cortical type

\begin{tabular}{|c|c|c|}
\hline Trajectory & Region & Cortical type \\
\hline \multirow[t]{7}{*}{ Linear } & Piriform & Allocortex \\
\hline & L entorhinal/perirhinal & Transition cortex \\
\hline & Subgenual cingulate & Transition cortex \\
\hline & Posterior orbitofrontal & Transition cortex \\
\hline & Frontal operculum & Transition cortex \\
\hline & Anterior insula & Transition cortex \\
\hline & Medial occipitotemporal & Homotypical isocortex \\
\hline \multirow[t]{7}{*}{ Quadratic } & Anterior cingulate (ventral supracallosal part) & Transition cortex \\
\hline & Posterior orbitofrontal & Transition cortex \\
\hline & Rentorhinal/perirhinal & Transition cortex \\
\hline & R parahippocampal & Homotypical isocortex \\
\hline & R anterior superior temporal & Homotypical isocortex \\
\hline & L temporal polar & Homotypical isocortex \\
\hline & Body of insula & Homotypical and dysgranular isocortex \\
\hline \multirow[t]{12}{*}{ Cubic } & Lateral orbitofrontal & Homotypical isocortex \\
\hline & Medial and lateral frontal pole & Homotypical isocortex \\
\hline & Lateral prefrontal (superior, middle, and inferior gyri) & Homotypical isocortex \\
\hline & Anterior cingulate (dorsal supracallosal part) & Agranular isocortex \\
\hline & Precentral motor & Agranular isocortex \\
\hline & Somatosensory & Granular isocortex \\
\hline & Posterior parietal & Homotypical isocortex \\
\hline & Posterior insular & Homotypical isocortex \\
\hline & Auditory & Granular isocortex \\
\hline & Lateral temporal cortex & Homotypical isocortex \\
\hline & Polar occipital & Granular isocortex \\
\hline & Lateral occipital (superior, middle, and inferior gyri) & Homotypical isocortex \\
\hline
\end{tabular}

Homotypical isocortex corresponds to the six layer prototype described by Brodmann; the granular cortex is similar to the homotypical isocortex, but has a thick and sometimes complex internal granular layer (layer 4); the agranular isocortex develops in the six-layer pattern of homotypical isocortex, but the cells composing layer 4 disperse during development; dysgranular isocortex has a thin, but discernable layer 4 and in that sense in intermediate between agranular isocortex and homotypical isocortex (but it is not, however, intermediate between isocortex and allocortex); allocortex, the three-layer cortex typified in mammalian brains by the hippocampus and piriform area; transition cortex, cortical areas, typically limbic, with a laminar organization intermediate between allocortex and isocortex. L, Left; $R$, right.

experience as one determinant of cortical architecture. Our assessment of the environmental factors was limited to a child's socioeconomic status and entering this measure as a covariate did not change the pattern of results. It would however be interesting to examine the impact of other key factors, particularly family and school environments on cortical development. Individual differences in intelligence influence cortical thickness and its development (Narr et al., 2006; Shaw et al., 2006b). However, our principal findings held when IQ was entered as a covariate, implying that although intelligence may influence some properties of cortical growth trajectories, such as velocity and the age of attaining peak cortical thickness, it does not impact on the basic link between complexity of cytoarchitecture and complexity of developmental trajectory.

Genetic factors are also important in determining cortical architecture (Thompson et al., 2001; Lenroot et al., 2007). Common polymorphisms such as the catechol-O-methyltransferase Val158Met polymorphism, a single nucleotide polymorphism in the regulator of G-protein signaling 4 gene, and a promoter region polymorphism of the serotonin transporter gene (5-HTTLPR) have all been found to have some influence on cortical volume, thickness or complexity (Brown and Hariri, 2006; Meyer-Lindenberg et al., 2006; Zinkstok et al., 2006; Buckholtz et al., 2007; Taylor et al., 2007). Of particular interest are genes that both contribute to both cortical growth and complexity and appear to be under positive selection in primate evolution, particularly in lineages leading to modern humans (Gilbert et al., 2005). These include the ASPM (abnormal spindle-like, microcephaly associated) and MCPH1 (microcephaly, primary autosomal recessive) genes (Evans et al., 2004a,b). It would be interesting to determine whether variation in the regional cortical expression of such genes aligns with both cortical types and developmental trajectory maps.

\section{Functional considerations}

Detailed consideration of these developmental patterns and their possible relationship to cognitive development remains for future work, but a few points can be made. For example, the posterior medial orbitofrontal areas have been linked with the limbic system and control of the autonomic nervous system, and they show a linear trajectory. These areas are thought to monitor the outcomes associated with behavior, particularly punishment or reward (Rolls, 2004; Kennerley et al., 2006), cognitive functions so fundamental that they are unlikely to undergo prolonged development. In contrast, isocortical regions often support more complex psychological functions, which show clear developmental gradients, characterized by rapid development during critical periods. We can only speculate about a possible relationship between critical periods for human skill development and the developmental trajectories described here. The delineation of critical periods for human skill development is complex, but late childhood is a period of particularly rapid development of executive skills of planning, working memory and cognitive flexibility, an age period which coincides with an increase in cortical thickness in the lateral prefrontal cortex (Chelune and Baer, 1986; Diamond, 2002; Huizinga et al., 2006; Jacobs et al., 2007). In contrast, the critical period for certain visual functions (such as letter acuity and global motion detection) has been estimated as ending in middle childhood ( age 6 or 7 ) (Lewis and Maurer, 2005) and, likewise, the period of increase in cortical thickness in the visual cortex also ends around this time (approximately ages 7-8). This correlation between the duration of some critical periods with the phase of increase in cortical thickness is certainly not universal. It is necessarily limited by the existence of systems (supported by similar cortical regions) with multiple critical periods, each having a different temporal window, as occurs in certain sensory systems. (Harrison et al., 2005; Levi, 2005; Lewis and Maurer, 2005). This discussion focuses on critical periods and should not be taken as dismissing the important of continued refinement of many cognitive skills during the adolescent phase of cortical thinning (Luna et al., 2004; Luciana et al., 2005).

\section{Conclusion}

The findings reported here support the idea that the organization of the cerebral cortex can be understood in terms of a series of concentric rings, with the isocortex (having a cubic trajectory) at its core, the allocortex (showing predominately linear growth) at the periphery, and the transitional areas (having a mix of quadratic and linear trajectories) in between. The isocortex in this model not only lies at the core of the cerebral cortex in this sense, but also arises later in evolution than the piriform area (lateral allocortex) and hippocampus (medial allocortex), and additional 

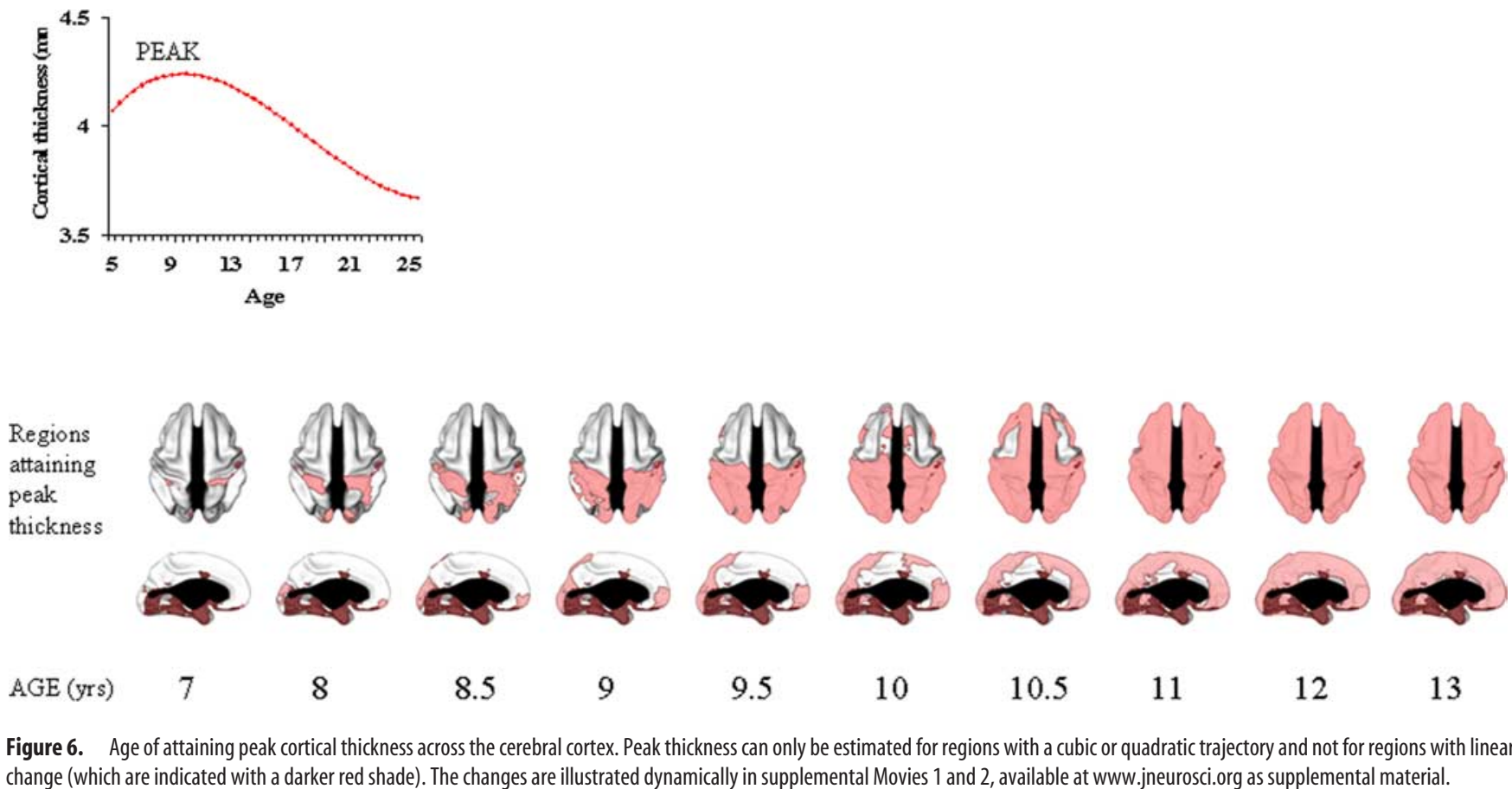

small allocortical areas. Thus, through in vivo neuroanatomic imaging we demonstrate that cortical development mirrors both the cytoarchitecture and history of the cerebral cortex.

\section{References}

Allman J, Hakeem A, Watson K (2002) Two phylogenetic specializations in the human brain. Neuroscientist 8:335-346.

Brockhaus H (1940) Die cyto- und myleoarchitcktonik des crotex clastralis und des clastrum beim menschen. J Psychol Neurol 49:249-348.

Brown SM, Hariri AR (2006) Neuroimaging studies of serotonin gene polymorphisms: exploring the interplay of genes, brain, and behavior. Cogn Affect Behav Neurosci 6:44-52.

Buckholtz JW, Meyer-Lindenberg A, Honea RA, Straub RE, Pezawas L, Egan MF, Vakkalanka R, Kolachana B, Verchinski BA, Sust S, Mattay VS, Weinberger DR, Callicott JH (2007) Allelic variation in RGS4 impacts functional and structural connectivity in the human brain. J Neurosci 27:1584-1593.

Chelune GJ, Baer RA (1986) Developmental norms for the Wisconsin Card Sorting test. J Clin Exp Neuropsychol 8:219-228.

Chklovskii DB, Mel BW, Svoboda K (2004) Cortical rewiring and information storage. Nature 431:782-788.

Diamond A (2002) Normal development of prefrontal cortex from birth to young adulthood: cognitive functions, anatomy and biochemistry. In: Principles of frontal lobe function (Stuss DT, Knight RT, eds), pp 466503. New York: Oxford UP.

Evans PD, Anderson JR, Vallender EJ, Choi SS, Lahn BT (2004a) Reconstructing the evolutionary history of microcephalin, a gene controlling human brain size. Hum Mol Genet 13:1139-1145.

Evans PD, Anderson JR, Vallender EJ, Gilbert SL, Malcom CM, Dorus S, Lahn BT (2004b) Adaptive evolution of ASPM, a major determinant of cerebral cortical size in humans. Hum Mol Genet 13:489-494.

Giedd JN, Snell JW, Lange N, Rajapakse JC, Casey BJ, Kozuch PL, Vaituzis AC, Vauss YC, Hamburger SD, Kaysen D, Rapoport JL (1996) Quantitative magnetic resonance imaging of human brain development: ages 4-18. Cereb Cortex 6:551-560.

Giedd JN, Blumenthal J, Jeffries NO, Castellanos FX, Liu H, Zijdenbos A, Paus T, Evans AC, Rapoport JL (1999) Brain development during childhood and adolescence: a longitudinal MRI study. Nat Neurosci 2:861-863.

Gilbert SL, Dobyns WB, Lahn BT (2005) Genetic links between brain development and brain evolution. Nat Rev Genet 6:581-590.

Gogtay N, Giedd JN, Lusk L, Hayashi KM, Greenstein D, Vaituzis AC, Nugent III TF, Herman DH, Clasen LS, Toga AW, Rapoport JL, Thompson PM
(2004) Dynamic mapping of human cortical development during childhood through early adulthood. Proc Natl Acad Sci USA 101:8174-8179. Gogtay N, Nugent III TF, Herman DH, Ordonez A, Greenstein D, Hayashi KM, Clasen L, Toga AW, Giedd JN, Rapoport JL, Thompson PM (2006) Dynamic mapping of normal human hippocampal development. Hippocampus 16:664-672.

Harrison RV, Gordon KA, Mount RJ (2005) Is there a critical period for cochlear implantation in congenitally deaf children? Analyses of hearing and speech perception performance after implantation. Dev Psychobiol 46:252-261.

Hensch TK (2004) Critical period regulation. Annu Rev Neurosci 27:549-579.

Hensch TK (2005) Critical period plasticity in local cortical circuits. Nat Rev Neurosci 6:877-888.

Hollingshead AB (1975) Four-factor index for social status. New Haven, CT: Yale UP.

Huizinga M, Dolan CV, van der Molen MW (2006) Age-related change in executive function: developmental trends and a latent variable analysis. Neuropsychologia 44:2017-2036.

Huttenlocher PR, Dabholkar AS (1997) Regional differences in synaptogenesis in human cerebral cortex. J Comp Neurol 387:167-178.

Jacobs R, Harvey AS, Anderson V (2007) Executive function following focal frontal lobe lesions: impact of timing of lesion on outcome. Cortex 43:792-805.

Jolicoeur P, Pontier J, Pernin MO, Sempe M (1988) A lifetime asymptotic growth curve for human height. Biometrics 44:995-1003.

Kaas JH (1987) The organization of neocortex in mammals: implications for theories of brain function. Annu Rev Psychol 38:129-151.

Kabani N, Le Goualher G, MacDonald D, Evans AC (2001) Measurement of cortical thickness using an automated 3-D algorithm: a validation study. NeuroImage 13:375-380

Kennerley SW, Walton ME, Behrens TE, Buckley MJ, Rushworth MF (2006) Optimal decision making and the anterior cingulate cortex. Nat Neurosci 9:940-947.

Knudsen EI (2004) Sensitive periods in the development of the brain and behavior. J Cogn Neurosci 16:1412-1425.

Kostovic I, Rakic P (1990) Developmental history of the transient subplate zone in the visual and somatosensory cortex of the macaque monkey and human brain. J Comp Neurol 297:441-470.

Kostovic I, Judas M, Rados M, Hrabac P (2002) Laminar organization of the human fetal cerebrum revealed by histochemical markers and magnetic resonance imaging. Cereb Cortex 12:536-544. 
Table 2. The estimated age of peak cortical thickness is given for 56 brain regions

\begin{tabular}{|c|c|c|c|}
\hline & Hemisphere & Trajectory & $\begin{array}{l}\text { Age of peak cortical } \\
\text { thickness (years) }\end{array}$ \\
\hline \multicolumn{4}{|l|}{ Frontal } \\
\hline \multirow[t]{2}{*}{ Superior } & $\mathrm{R}$ & Cubic & 10.2 \\
\hline & $\mathrm{L}$ & Cubic & 10.1 \\
\hline \multirow[t]{2}{*}{ Middle } & $\mathrm{R}$ & Cubic & 10.4 \\
\hline & $\mathrm{L}$ & Cubic & 10.3 \\
\hline \multirow[t]{2}{*}{ Inferior } & $\mathrm{R}$ & Cubic & 9.7 \\
\hline & $\mathrm{L}$ & Cubic & 9.7 \\
\hline \multirow[t]{2}{*}{ Medial } & $\mathrm{R}$ & Cubic & 10.6 \\
\hline & $\mathrm{L}$ & Cubic & 10.0 \\
\hline \multirow[t]{2}{*}{ Precentral } & $\mathrm{R}$ & Cubic & 9.6 \\
\hline & $\mathrm{L}$ & Cubic & 10.5 \\
\hline \multirow{2}{*}{ Cingulate } & $\mathrm{R}$ & Cubic & 13.8 \\
\hline & $\mathrm{L}$ & Cubic & 11.2 \\
\hline \multirow[t]{2}{*}{ Medial orbitofrontal } & $\mathrm{R}$ & Linear & \\
\hline & $\mathrm{L}$ & Cubic & 8.6 \\
\hline \multirow[t]{2}{*}{ Lateral orbitofrontal } & $\mathrm{R}$ & Cubic & 9.4 \\
\hline & $\mathrm{L}$ & Cubic & 9.4 \\
\hline \multicolumn{4}{|l|}{ Temporal } \\
\hline \multirow[t]{2}{*}{ Superior } & $\mathrm{R}$ & Cubic & 14.9 \\
\hline & $\mathrm{L}$ & Cubic & 14.9 \\
\hline \multirow[t]{2}{*}{ Middle } & $\mathrm{R}$ & Cubic & 11.7 \\
\hline & $\mathrm{L}$ & Cubic & 11.6 \\
\hline \multirow{2}{*}{ Inferior } & $\mathrm{R}$ & Cubic & 11.2 \\
\hline & $\mathrm{L}$ & Cubic & 11.1 \\
\hline \multirow[t]{2}{*}{ Insula } & $\mathrm{R}$ & Quadratic & 18.1 \\
\hline & $\mathrm{L}$ & Quadratic & 18.0 \\
\hline \multirow[t]{2}{*}{ Periamygdaloid } & $\mathrm{R}$ & Linear & \\
\hline & $\mathrm{L}$ & Linear & \\
\hline \multirow[t]{2}{*}{ Parahippocampal } & $\mathrm{R}$ & Linear & \\
\hline & $\mathrm{L}$ & Linear & \\
\hline \multirow[t]{2}{*}{ Uncal } & $\mathrm{R}$ & Linear & \\
\hline & $L$ & Linear & \\
\hline Parietal & & & \\
\hline Postcentral gyrus & $\mathrm{R}$ & Cubic & 8.4 \\
\hline & $\mathrm{L}$ & Cubic & 8.5 \\
\hline Superior lobule & $\mathrm{R}$ & Cubic & 8.3 \\
\hline & $\mathrm{L}$ & Cubic & 9.0 \\
\hline Supramarginal gryus & $\mathrm{R}$ & Cubic & 9.2 \\
\hline & $\mathrm{L}$ & Cubic & 9.2 \\
\hline Angular gryus & $\mathrm{R}$ & Cubic & 8.5 \\
\hline & $\mathrm{L}$ & Cubic & 9.0 \\
\hline Precuneus & $\mathrm{R}$ & Cubic & 9.8 \\
\hline & $\mathrm{L}$ & Cubic & 10.1 \\
\hline Lateral occipitotemporal & $\mathrm{R}$ & Cubic & 11.2 \\
\hline & $\mathrm{L}$ & Cubic & 11.1 \\
\hline Medial occipitotemporal & $\mathrm{R}$ & Linear & \\
\hline & $\mathrm{L}$ & Linear & \\
\hline Occipital & & & \\
\hline Pole & $\mathrm{R}$ & Cubic & 7.9 \\
\hline & $\mathrm{L}$ & Cubic & 6.8 \\
\hline Superior & $\mathrm{R}$ & Cubic & 8.3 \\
\hline & $\mathrm{L}$ & Cubic & 8.3 \\
\hline Middle & $\mathrm{R}$ & Cubic & 9.5 \\
\hline & $\mathrm{L}$ & Cubic & 9.2 \\
\hline Inferior & $\mathrm{R}$ & Cubic & 7.3 \\
\hline & $\mathrm{L}$ & Cubic & 7.9 \\
\hline Lingual & $\mathrm{R}$ & Cubic & 8.6 \\
\hline & $\mathrm{L}$ & Cubic & 9.2 \\
\hline Cuneus & $\mathrm{R}$ & Cubic & 9.2 \\
\hline & $\mathrm{L}$ & Cubic & 8.8 \\
\hline
\end{tabular}

This can be estimated only for regions with either a cubic of quadratic trajectory (thus, regions with a linear trajectory are marked with a dash). For regions with a mix of trajectories (e.g., the insula), the dominant trajectory, which applies to the majority of points within the region, is given. $L$, Left; $R$, right.
Lee JK, Lee JM, Kim JS, Kim IY, Evans AC, Kim SI (2006) A novel quantitative cross-validation of different cortical surface reconstruction algorithms using MRI phantom. NeuroImage 31:572-584.

Lenroot RK, Schmitt JE, Ordaz SJ, Wallace GL, Neale MC, Lerch JP, Kendler KS, Evans AC, Giedd JN (2007) Differences in genetic and environmental influences on the human cerebral cortex associated with development during childhood and adolescence. Hum Brain Mapp, in press.

Lerch JP, Evans AC (2005) Cortical thickness analysis examined through power analysis and a population simulation. NeuroImage 24:163-173.

Lerch JP, Pruessner JC, Zijdenbos A, Hampel H, Teipel SJ, Evans AC (2005) Focal decline of cortical thickness in Alzheimer's disease identified by computational neuroanatomy. Cereb Cortex 15:995-1001.

Levi DM (2005) Perceptual learning in adults with amblyopia: a reevaluation of critical periods in human vision. Dev Psychobiol 46:222-232.

Lewis TL, Maurer D (2005) Multiple sensitive periods in human visual development: evidence from visually deprived children. Dev Psychobiol 46:163-183.

Lu LH, Leonard CM, Thompson PM, Kan E, Jolley J, Welcome SE, Toga AW, Sowell ER (2007) Normal developmental changes in inferior frontal gray matter are associated with improvement in phonological processing: a longitudinal MRI analysis. Cereb Cortex 17:1092-1099.

Luciana M, Conklin HM, Hooper CJ, Yarger RS (2005) The development of nonverbal working memory and executive control processes in adolescents. Child Dev 76:697-712.

Luna B, Garver KE, Urban TA, Lazar NA, Sweeney JA (2004) Maturation of cognitive processes from late childhood to adulthood. Child Development 75:1357-1372.

MacDonald D (1996) MNI-display. Montreal: McConnell Brain Imaging Center, Montreal Neurological Institute.

MacDonald D, Kabani N, Avis D, Evans AC (2000) Automated 3-D extraction of inner and outer surfaces of cerebral cortex from MRI. NeuroImage 12:340-356.

Makris N, Biederman J, Valera EM, Bush G, Kaiser J, Kennedy DN, Caviness VS, Faraone SV, Seidman LJ (2006) Cortical thinning of the attention and executive function networks in adults with attention-deficit/hyperactivity disorder. Cereb Cortex 17:1364-1375.

Mataga N, Mizuguchi Y, Hensch TK (2004) Experience-dependent pruning of dendritic spines in visual cortex by tissue plasminogen activator. Neuron 44:1031-1041.

Mesulam MM, Mufson EJ (1982) Insula of the old world monkey. I. Architectonics in the insulo-orbito-temporal component of the paralimbic brain. J Comp Neurol 212:1-22.

Meyer-Lindenberg A, Nichols T, Callicott JH, Ding J, Kolachana B, Buckholtz J, Mattay VS, Egan M, Weinberger DR (2006) Impact of complex genetic variation in COMT on human brain function. Mol Psychiatry 11:867-877.

Narr KL, Woods RP, Thompson PM, Szeszko P, Robinson D, Dimtcheva T, Gurbani M, Toga AW, Bilder RM (2006) Relationships between IQ and regional cortical gray matter thickness in healthy adults. Cereb Cortex 17:2163-2171.

O’Donnell S, Noseworthy MD, Levine B, Dennis M (2005) Cortical thickness of the frontopolar area in typically developing children and adolescents. NeuroImage 24:948-954.

Ongur D, Ferry AT, Price JL (2003) Architectonic subdivision of the human orbital and medial prefrontal cortex. J Comp Neurol 460:425-449.

Pinheiro JC, Bates DM (2000) Mixed-effects models in S and S-PLUS. New York: Springer.

Puelles L (2001) Thoughts on the development, structure and evolution of the mammalian and avian telencephalic pallium. Philos Trans R Soc Lond B Biol Sci 356:1583-1598.

Rolls ET (2004) The functions of the orbitofrontal cortex. Brain Cogn 55:11-29.

Shaw P, Lerch J, Greenstein D, Sharp W, Clasen L, Evans A, Giedd J, Castellanos FX, Rapoport J (2006a) Longitudinal mapping of cortical thickness and clinical outcome in children and adolescents with attentiondeficit/hyperactivity disorder. Arch Gen Psychiatry 63:540-549.

Shaw P, Greenstein D, Lerch J, Clasen L, Lenroot R, Gogtay N, Evans A, Rapoport J, Giedd J (2006b) Intellectual ability and cortical development in children and adolescents. Nature 440:676-679.

Sled JG, Zijdenbos AP, Evans AC (1998) A nonparametric method for automatic correction of intensity nonuniformity in MRI data. IEEE Trans Med Imaging 17:87-97. 
Sowell ER, Thompson PM, Leonard CM, Welcome SE, Kan E, Toga AW (2004) Longitudinal mapping of cortical thickness and brain growth in normal children. J Neurosci 24:8223-8231.

Sowell ER, Peterson BS, Kan E, Woods RP, Yoshii J, Bansal R, Xu D, Zhu H, Thompson PM, Toga AW (2007) Sex differences in cortical thickness mapped in 176 healthy individuals between 7 and 87 years of age. Cereb Cortex 17:1550-1560.

Striedter GF (2005) Principles of brain evolution. Sunderland, MA: Sinauer.

Sur M, Rubenstein JL (2005) Patterning and plasticity of the cerebral cortex. Science 310:805-810.

Tanner JM, Whitehouse RH, Marubini E, Resele LF (1976) The adolescent growth spurt of boys and girls of the Harpenden growth study. Ann Hum Biol 3:109-126.

Taylor WD, Zuchner S, Payne ME, Messer DF, Doty TJ, MacFall JR, Beyer JL, Krishnan KRR (2007) The COMT Val158Met polymorphism and temporal lobe morphometry in healthy adults. Psychiatry Res 155:173-177.

Thompson PM, Cannon TD, Narr KL, van Erp T, Poutanen VP, Huttunen M,
Lonnqvist J, Standertskjold-Nordenstam CG, Kaprio J, Khaledy M, Dail R, Zoumalan CI, Toga AW (2001) Genetic influences on brain structure. Nature Neuroscience 4:1253-1258.

von Economo C, Koskinas GN (1925) Die dytoarchitektonik der hirnrinde des erwachsenen menschen. Berlin: Springer.

Yakovlev PI, Lecours AR (1967) The myelinogenetic cycles of regional maturation of the brain. In: Regional development of the brain in early life (Minokowski A, ed). Oxford: Blackwell Scientific.

Zijdenbos AP, Forghani R, Evans AC (2002) Automatic "pipeline" analysis of 3-D MRI data for clinical trials: application to multiple sclerosis. IEEE Trans Med Imaging 21:1280-1291.

Zilles K, Palomero-Gallagher N, Schleicher A (2004) Transmitter receptors and functional anatomy of the cerebral cortex. J Anat 205:417-432.

Zinkstok J, Schmitz N, van Amelsvoort T, de Win M, van den Brink W, Baas F, Linszen D (2006) The COMT val158met polymorphism and brain morphometry in healthy young adults. Neurosci Lett 405:34-39. 